\title{
Hyaluronidase Coated Molecular Envelope Technology Nanoparticles Enhance Drug Absorption via the Subcutaneous Route
}

\author{
Ramesh Soundararajan, George Wang, Asya Petkova, Ijeoma F. Uchegbu, and Andreas G. Schätzlein*
}

Cite This: https://dx.doi.org/10.1021/acs.molpharmaceut.0c00294

(5) Read Online

ACCESS | W W Metrics \& More 回 Article Recommendations
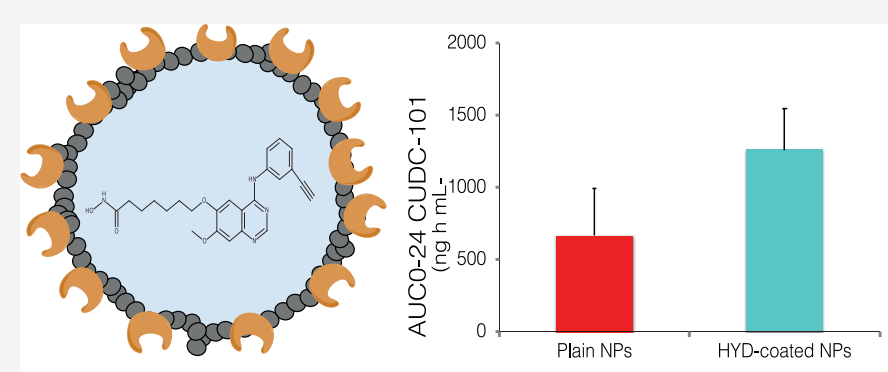

CUDC101 in Subcutaneous

Polymeric Nanoparticles

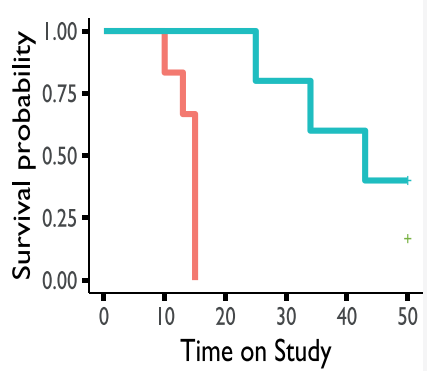

Therapeutic effect

ABSTRACT: Parenteral chemotherapy is usually administered intravenously, although patient preference and health economics suggest the subcutaneous (sc) route could be an attractive alternative. However, due to the low aqueous solubility of hydrophobic drugs and injection volume limitations, the total amount of drug that can be administered in a single sc injection is frequently insufficient. We have developed hyaluronidase coated nanoparticles (NPs) that efficiently encapsulate such drugs, thus addressing both issues and allowing sufficient amounts of hydrophobic drug to be administered and absorbed effectively. CUDC-101, a poorly water-soluble multitargeted anticancer drug that simultaneously inhibits the receptor tyrosine kinases (RTKs) EGFR and HER2, as well as histone deacetylase (HDAC), was encapsulated in polymeric Molecular Envelope Technology (MET) NPs. The role of polymer chemistry, formulation parameters, and coating with hyaluronidase (HYD) on MET-CUDC-101 NP formulations was examined and optimized to yield high drug loading and colloidal stability, and, after freeze-drying, stable storage at room temperature for up to 90 days. The pharmacokinetic studies in healthy rats showed that plasma $\mathrm{AUC}_{0-24 \mathrm{~h}}$ after sc administration correlates tightly with formulation physical chemistry, specifically in vitro colloidal stability. Compared to uncoated NPs, the HYDcoating doubled the drug plasma exposure. In a murine A431 xenograft model, the coated HYD-MET-CUDC-101 NPs at a dose equivalent to $90 \mathrm{mg} \mathrm{kg}^{-1}$ CUDC-101 increased the survival time from 15 days (control animals treated with hyaluronidase alone) to 43 days. Polymer MET nanoparticles coated with hyaluronidase enabled the subcutaneous delivery of a hydrophobic drug with favorable therapeutic outcomes.

KEYWORDS: hyaluronidase, subcutaneous administration, polymeric micelles, CUDC-101, murine xenograft tumor

\section{INTRODUCTION}

Many drugs, in particular in cancer therapy, routinely utilize the intravenous route of administration, although the subcutaneous route of administration might actually be more advantageous. For example, recent studies show that subcutaneous administration is as safe and effective as intravenous injection/infusion for the administration of bortezomib $^{1}$ or desmopressin (DDAVP). ${ }^{2}$ Patients generally prefer the subcutaneous route of administration over the intravenous route, ${ }^{3}$ and, crucially, subcutaneous formulations may be administered in the home.
However, the volume that can be administered subcutaneously is typically limited to below $2 \mathrm{~mL}$ due to the mechanical resistance in the tissue. ${ }^{4}$ The subcutaneous space consists of adipose tissue, connective tissue containing an extracellular matrix (ECM) consisting of collagen, hyaluronic

Received: March 18, 2020

Revised: May 6, 2020

Accepted: May 7, 2020

Published: May 7, 2020 
acid, and other glycosaminoglycans such as chrondroitin-4sulfate and chrondroitin-6-sulfate. On subcutaneous administration of drugs this combination of cells and extracellular matrix limits the spread and volume that can be accommodated in the tissue as well as the absorption from the tissue into the capillaries and lymphatic vessels. ${ }^{5}$

Hyaluronidase (HYD) has been used as a spreading agent for the subcutaneous route for over 50 years, enabling volumes in excess of the normal $2 \mathrm{~mL}^{4}$ to be administered due to a loosening of the connective tissue by enzymatic cleavage of ECM biopolymer components. ${ }^{6}$ Specifically, HYD (whether of bacterial or vertebral in origin) catalyzes the hydrolysis of hyaluronic acid at the 1,4 glycosidic linkages. ${ }^{7}$ Vertebrate HYD, as has been used in the current study (endo- $\beta$-acetylhexosaminidase), also catalyzes the degradation of chrondroitin and chrondroitin-6-sulfate at the 1,4 glycosidic linkage, albeit at a slower rate than for hyaluronic acid. ${ }^{7}$ Furthermore, subcutaneous formulations containing HYD that allow rapid injection of increased dose volumes of $5 \mathrm{~mL}$ of trastuzumab have recently been approved for human use ${ }^{8}$ and, more recently, up to more than $10 \mathrm{~mL}$ for subcutaneous rituximab. ${ }^{9}$

The wider application of HYD-based formulations is restricted, however, as it is usually applied for drugs with good water solubility that allows high concentrations and thus sufficient amounts to of drug be injected. Our goal was to extend the use of HYD to the delivery of hydrophobic drugs. This is important as $40 \%$ of approved drugs are practically insoluble in water (water solubility of $<0.1 \mathrm{mg} \mathrm{mL}^{-1}$ ). ${ }^{10}$ Poorly water-soluble drugs are often administered with organic solvents and surfactants, ${ }^{11}$ which may prove incompatible with HYD. We hypothesized that nanoparticles (NPs) ${ }^{12,13}$ could be used in order to facilitate the combination of hydrophobic drug and water-soluble enzyme: NPs would facilitate encapsulation of the poorly water-soluble drug at the required high dose in an aqueous volume. HYD may then be incorporated into the aqueous formulation either in the disperse phase or coated onto the drug encapsulating NPs. We hypothesized that the degradation of the extracellular matrix by particle bound hyaluronidase would retain a localized high enzymatic activity and should improve homogeneous drug absorption as well as enable a higher injection volume, and thus a higher dose of drug, to be administered via the subcutaneous route.

CUDC-101 is a novel multitarget inhibitor of histone deacetylase (HDAC), epidermal growth factor receptor (EGFR), and human epidermal growth factor receptor 2 (HER2). ${ }^{14}$ The compound is extremely hydrophobic (water solubility of $\sim 0.03 \mathrm{mg} \mathrm{mL}^{-1}$ ), and in the phase I clinical trial of CUDC-101 in head and neck squamous carcinoma patients the low oral bioavailability and need for alternative administration routes were identified. ${ }^{15}$ When administered intravenously, CUDC-101 is administered at a dose of 225$275 \mathrm{mg} \mathrm{m}^{2}$ with each patient requiring approximately 400$500 \mathrm{mg}$ of drug as a single dose. ${ }^{15}$

CUDC-101 was studied as a model for the subcutaneous route of administration as it presents the perfect set of challenges: the need to deliver a high dose $(500 \mathrm{mg})$, a highly hydrophobic nature (water solubility of $\sim 0.03 \mathrm{mg} \mathrm{mL}^{-1}$ ), coupled with the low liquid volume typically used for the subcutaneous administration (maximum human subcutaneous dose volume without hyaluronidase $=1.5 \mathrm{~mL}$ ). We chose to address these challenges using (a) $\mathrm{N}$-palmitoyl- $\mathrm{N}$-monomethyl-N,N-dimethyl-N,N,N-trimethyl-6-O-glycolchitosan $\mathrm{NPs}^{13}$ [also known as Molecular Envelope Technology (MET)] to incorporate sufficient concentration of the drug into the aqueous volume and (b) a coating of these NPs with HYD in order to facilitate the absorption of drug from these polymeric NPs, via the subcutaneous route and from a high dose volume. The effect of polymer chemistry and formulation on NP physical chemistry, resulting drug pharmacokinetics, and therapeutic efficacy were then studied.

\section{EXPERIMENTAL SECTION}

All chemicals were supplied by Sigma-Aldrich (Dorset, U.K.) unless otherwise specified. CUDC-101 was supplied by Curis Inc. (Boston, MA, USA). N-Palmitoyl- $N$-monomethyl- $N, N$ dimethyl-N,N,N-trimethyl-6-O-glycolchitosan [molecular envelope technology (MET)] was supplied by Nanomerics Ltd. (London, U.K.). Dextran $6 \mathrm{kDa}$ was supplied by Alfa Aesar (MA, USA). Organic solvents were supplied by the UCL School of Pharmacy (London, U.K.). Visking seamless cellulose dialysis membranes were obtained from Medicell International Ltd. (London, U.K.). All chemicals and reagents were used without further purification.

Preparation of MET-CUDC-101 Formulations. Three formulations were prepared that differed in the degree of palmitoylation (DP \%) and quaternization (DQ\%).

Formulation F1. CUDC-101 (50 mg) was dissolved in a solution of polyvinylpyrrolidone (1\% w/v, PVPK30) in $\mathrm{NaOH}$ $(0.2 \mathrm{M}, 5 \mathrm{~mL})$ by heating in a shaking water bath at $70{ }^{\circ} \mathrm{C}$ for 5 min. MET1 (100 mg, lot number GCPQ48070313VL, mole $\%$ palmitoyl groups $=14 \%$, mole $\%$ quaternary ammonium groups $=26 \%, \mathrm{Mw}=9210 \mathrm{Da}, \mathrm{Mn}=9180 \mathrm{Da}, \mathrm{Mw} / \mathrm{Mn}=$ 1.01) was dispersed in hydrochloric acid $(0.2 \mathrm{M}, 5 \mathrm{~mL})$ by bath sonication. To this MET1 dispersion was added the warm alkaline solution (clear yellow in color) of CUDC-101, and the resultant colloid suspension was vortexed for $10 \mathrm{~s}$. The $\mathrm{pH}$ of the resulting formulation was adjusted to $\mathrm{pH}=6.8$ by dropwise addition of sodium hydroxide solution (1 M).

Formulation F2. CUDC-101 (50 mg) was dissolved in $\mathrm{NaOH}(0.2 \mathrm{M}, 5 \mathrm{~mL})$ by heating in a shaking water bath at 70 ${ }^{\circ} \mathrm{C}$ for $5 \mathrm{~min}$. MET2 (100 mg, lot number GCPQ48 240114 SR, mole $\%$ palmitoyl groups $=5 \%$, mole $\%$ quaternary ammonium groups $=5 \%, \mathrm{Mw}=10040 \mathrm{Da}, \mathrm{Mn}=9420 \mathrm{Da}$, $\mathrm{Mw} / \mathrm{Mn}=1.065)$ was dispersed in water $(5 \mathrm{~mL})$ by shaking, yielding a slightly acidic nanoparticle suspension. To this MET2 suspension a warm alkaline solution (clear yellow in color) of CUDC-101 was added, and the resultant colloid suspension was vortexed for $10 \mathrm{~s}$. The $\mathrm{pH}$ of the resulting formulation was adjusted to $\mathrm{pH} \sim 6.8$ with $\mathrm{NaOH}(1 \mathrm{M})$. The freshly prepared formulation was imaged by transmission electron microscopy.

Formulation F3. CUDC-101 (50 mg) was dissolved in $\mathrm{NaOH}(0.2 \mathrm{M}, 1 \mathrm{~mL})$ by heating at $70{ }^{\circ} \mathrm{C}$. MET2 $(50 \mathrm{mg})$ was dispersed in water $(9 \mathrm{~mL})$ by shaking, and the warm solution of the drug was then added to the MET2 dispersion. The $\mathrm{pH}$ of the formulation was adjusted to $\mathrm{pH}=6.8$ using $\mathrm{NaOH}$ (1 $\mathrm{M})$, if necessary.

Concentrated Formulations F1-F3. Formulations containing higher drug concentrations for in vivo administration were achieved by reconstitution of freeze-dried material in a reduced volume (Table 1). Specifically, freshly prepared material was frozen in liquid nitrogen and then freeze-dried (over a $24 \mathrm{~h}$ period). For F3, dextran was added as a stabilizer $(100 \mathrm{mg}, 6 \mathrm{kDa})$ prior to freeze-drying. The freeze-dried cake was then reconstituted to $2 \mathrm{~mL}$ (formulations F1 and F2, 25 
Table 1. MET-CUDC-101 Formulations

\begin{tabular}{llll} 
& \multicolumn{1}{c}{$\mathrm{F} 1$} & $\mathrm{~F} 2$ & $\mathrm{~F} 3$ \\
polymer DP:DQ\% & $14: 26$ & $5: 5$ & $5: 5$ \\
CUDC-101:MET & $5: 10$ & $5: 10$ & $5: 5$ \\
& Freeze-Drying & & \\
reconstitution $\left(\mathrm{mL}\right.$ of $\left.\mathrm{H}_{2} \mathrm{O}\right)$ & 2 & 2 & 1 \\
CUDC-101:MET & $25: 50$ & $25: 50$ & $50: 50$ \\
drug loading $(\% \mathrm{w} / \mathrm{w})$ & 33 & 33 & 50 \\
\hline
\end{tabular}

$\mathrm{mg} \mathrm{mL} \mathrm{m}^{-1}$ CUDC-101) or $1 \mathrm{~mL}$ (formulation F3, $50 \mathrm{mg} \mathrm{mL}^{-1}$ of CUDC-101), respectively, in water by shaking or bath sonication.

Hyaluronidase-Coated Formulation F3-HYD. The freeze-dried MET-CUDC-101 formulation F3 from above was surface-coated with the enzyme by simply reconstituting the freeze-dried cake with a solution of bovine hyaluronidase (600 IU mg ${ }^{-1}$, Sigma, Gillingham, U.K.) in water resulting in F3-HYD containing HYD-MET-CUDC-101 NPs. The surface coating was confirmed using transmission electron microscopy (TEM), $\zeta$ potential measurements, and dynamic light scattering.

For the dynamic light scattering studies to examine the extent of hyaluronidase coating on F3-HYD, F3-HYD formulations were prepared and analyzed as described below.

CUDC-101 $10 \mathrm{mg}$ was dissolved in $0.2 \mathrm{M} \mathrm{NaOH}(0.4 \mathrm{~mL})$ by heating at $70{ }^{\circ} \mathrm{C}$ for $10 \mathrm{~min}$. MET2 (10 mg, mole \% palmitoyl groups $=6 \%$, mole $\%$ quaternary ammonium groups $=6 \%, \mathrm{Mw}=10000 \mathrm{Da})$ was dispersed in water $(3.6 \mathrm{~mL})$ by shaking. $40 \mathrm{mg}$ of $6 \mathrm{kDa}$ dextran was added to the MET dispersion. The warm solution of the solubilized drug (light yellow) was then added to the MET2 dispersion containing dextran. The $\mathrm{pH}$ of the formulation was adjusted to $\mathrm{pH}=6.8$ using HCL. The formulation was then freeze-dried over $24 \mathrm{~h}$. The freeze-dried MET-CUDC-101 formulation F3 was coated with hyaluronidase by simply reconstituting the freeze-dried cake with a solution of bovine hyaluronidase $(1 \mathrm{~mL})$ at a variety of hyaluronidase concentrations $\left(0.3 \mathrm{mg} \mathrm{mL}^{-1}, 0.5 \mathrm{mg}\right.$ $\mathrm{mL}^{-1}, 1 \mathrm{mg} \mathrm{mL}^{-1}, 5 \mathrm{mg} \mathrm{mL}^{-1}$, and $\left.10 \mathrm{mg} \mathrm{mL}^{-1}\right)$.

Characterization of CUDC-101 Formulations. HPLC was performed using an Agilent 1200 series instrument with UV detection (Agilent Technologies, U.K.), and chromatography was carried out over two Onyx Monolithic C18 columns (each $100 \mathrm{~mm} \times 4.6 \mathrm{~mm}$, Phenomenex, U.K.) connected in series with an Onyx polymeric column coupler. The system was also equipped with an Onyx C18 guard column $(5 \mathrm{~mm} \times$ $4.6 \mathrm{~mm}$, pore size $=0.01 \mu \mathrm{m})$. A gradient method (Table 2) using a mixture of acetonitrile (ACN) and trifluoroacetic acid (TFA) was used to elute the analytes, and the flow rate was 2.0 $\mathrm{mL} \min ^{-1}$. The column temperature was maintained at $35^{\circ} \mathrm{C}$, and the detection wavelength was $254 \mathrm{~nm}$. The retention time for CUDC-101 was $13.1 \mathrm{~min}$. The injected volume was $20 \mu \mathrm{L}$.

Table 2. Gradient Method for CUDC-101 HPLC Analysis

$\begin{array}{ccc}\text { time }(\mathrm{min}) & \text { TFA (\%) } & \text { ACN }(\%) \\ 0 & 90 & 10 \\ 3 & 85 & 15 \\ 10 & 75 & 25 \\ 20 & 15 & 85 \\ 20.2 & 90 & 10 \\ 23.2 & 90 & 10\end{array}$

The drug content was quantified using a freshly prepared standard curve (Table 2).

CUDC-101 standard curves were prepared as described below. A solution of CUDC-101 $\left(10 \mathrm{mg} \mathrm{mL}^{-1}\right)$ in dimethyl sulfoxide was prepared, followed by dilution in acetonitrile to give a dilute CUDC-101 stock solution $\left(1 \mathrm{mg} \mathrm{mL}^{-1}\right)$.

Standard solutions $\left(1,10,25,50,100\right.$, and $\left.200 \mu \mathrm{g} \mathrm{mL}^{-1}\right)$ were prepared by diluting this stock solution $(1,10,25,50$, 100 , and $200 \mu \mathrm{L}$ respectively) to $1 \mathrm{~mL}$ in acetonitrile. Standard curve parameters from validation runs on different days are summarized in Table 3, demonstrating excellent interday reproducibility.

\section{Table 3. CUDC-101 HPLC Standard Curves ${ }^{a}$}

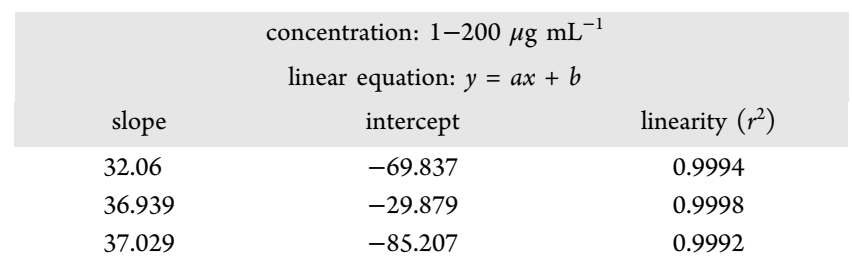

${ }^{a}$ CUDC-101 lower limit of quantification $=1 \mu \mathrm{g} \mathrm{mL}^{-1}$; CUDC-101 limit of detection $=100 \mathrm{ng} \mathrm{mL}{ }^{-1}$.

TEM was carried out by Philips/FEI CM120 Bio Twin (Philips, The Netherlands). A drop of the formulation was dried on a copper TEM grid (300 mesh, Formvar/carbon coated) and stained with a drop of uranyl acetate $(1 \% \mathrm{w} / \mathrm{v}$, negative staining). Once dried, the samples were analyzed under the TEM, and the representative images were photographed and documented.

The nanoparticles were also sized using dynamic light scattering. The viscous MET formulations were diluted 10 times in $\mathrm{NaCl}(10 \mathrm{mM})$ before the size measurement using the Malvern Zetasizer Nano ZS ZEN3600 (Malvern Instruments Ltd., Malvern, U.K.). The diluted formulations were dispersed into low volume dispersible cuvettes and the size distribution graphs recorded. Sizing was carried out using the Contin method of analysis. The $\zeta$ potential of the formulations was measured, as detailed above, and their morphology recorded using TEM, as detailed above.

Gel electrophoresis was carried out on the F3-HYD formulation containing $10 \mathrm{mg} \mathrm{mL} \mathrm{m}^{-1}$ hyaluronidase. The gel was removed from the pouch and rinsed with deionized water before assembly into the X-Cell Sure Lock gel electrophoresis system. The X-cell Sure Lock chamber was filled with Trisacetate running buffer to cover the wells of the gel. Samples were prepared in a total volume of $25 \mu \mathrm{L}$ and total protein amount of $30 \mu \mathrm{g}(12.5 \mu \mathrm{L}$ of Tris-glycine native sample buffer $2 \times)$. The gel was run for $1.5 \mathrm{~h}$ at $150 \mathrm{~V}$ constant voltage. Bands were developed using $20 \mathrm{~mL}$ of InstantBlue protein stain overnight before imaging.

Stability and NP Reconstitution Studies. The colloidal stability of nanoparticles was measured fresh or after dispersing the freeze-dried formulations in water and leaving them undisturbed at room temperature. Aliquots $(100 \mu \mathrm{L})$ were withdrawn at specific time points, centrifuged at $1000 \mathrm{~g}$ for 10 min (MSE Micro Centaur, London, U.K.), and the colloidal fraction (supernatant) was collected and analyzed for drug content. Long-term storage stability studies were carried out on freeze-dried MET-CUDC-101 formulation F3. For this, the freeze-dried formulation was stored at room temperature, 
samples were withdrawn at predetermined time points and reconstituted in water, and the colloidal fraction was analyzed, as described above.

The nanoparticles were also sized and $\zeta$ potential was measured using the methods described above. The viscous MET formulations were diluted 10 times in $\mathrm{NaCl}(10 \mathrm{mM})$ before the size measurement using the Malvern Zetasizer Nano ZS ZEN3600 (Malvern Instruments Ltd., Malvern, U.K.). The diluted formulations were dispersed into low volume dispersible cuvettes and the size distribution graphs recorded. Sizing was carried out using the Contin method of analysis. The $\zeta$ potential of the formulations was measured, as detailed above, and their morphology recorded using TEM, as detailed above.

X-ray diffraction (XRD) patterns of the freeze-dried METCUDC-101 formulation and relevant reference materials were obtained using a Miniflex 600 (Rigaku, Tokyo, Japan). Powdered formulations were filled into a hollow aluminum sample holder, and the X-ray diffraction patterns were recorded in the $2 \theta$ range $4-60^{\circ}$ at a speed of $5^{\circ}$ per min $\left(\right.$ step $\left.=0.02^{\circ}\right)$.

Pharmacokinetics Studies. All animal experiments were performed under license from the U.K. Home Office and in accordance with the U.K. Animal Scientific Procedures Act 1986. Male Wistar rats (Harlan, Oxon, U.K., 200-250 g in weight) were acclimatized for at least 5 days before the experiment, in the animal housing unit, maintained at an ambient temperature of $22{ }^{\circ} \mathrm{C}$, relative humidity of $60 \%$, and equal day-night cycle. The animals were given free access to food and water throughout the experiment. The CUDC-101 formulation was then injected subcutaneously on the thigh (1-2 mL of $10 \mathrm{~mL} \mathrm{~kg}{ }^{-1}$ per rat to achieve the same dose of CUDC-101, $n=4)$. Blood samples were collected from the rats at predetermined time points via tail-vein bleeding, and at the end of the experiment the rats were euthanized and blood was collected through cardiac puncture. EDTA blood samples were collected from the tail vein, and plasma was collected by centrifugation $\left(10 \mathrm{~min} 1000 \mathrm{~g}\right.$ ) and stored at $-80{ }^{\circ} \mathrm{C}$ unless analyzed immediately. For LC-MS/MS analysis plasma (50 $\mu \mathrm{L}$ ) was spiked with CUDC-101 internal standard (IS, $5 \mu \mathrm{L}$, $\left.250 \mathrm{ng} \mathrm{mL} \mathrm{m}^{-1}\right)$. Extraction into $3 \times$ volume of acetonitrile containing $0.1 \% \mathrm{FA}$ was achieved after mixing/vortexing and collection of supernatant $(10000 \mathrm{~g}$ for $10 \mathrm{~min})$.The subcutaneous injection site was examined for drug precipitation by removing the skin at the site and examining the site visually or examining any residue with a microscope.

Pharmacodynamics Studies. Female athymic nude mice (CD-1 nu/nu, 20-30 g) aged 6-8 weeks were obtained from Charles River Laboratories, Harlow, U.K. They were housed in ventilated microisolator cages in a controlled climate, fed irradiated laboratory rodent diet ad libitum, and provided sterilized water. All housing and supplies for nude mice were sterilized by autoclaving before use. Mice were inspected daily including weekends/holidays, and all animal procedures were performed under sterile conditions within a biosafety cabinet (for injections) or laminar flow hood (for animal husbandry and noninvasive procedures).

A431 (epidermoid carcinoma) cells were obtained from ATCC (American Type Culture Collection). Studies were carried out under biosafety level 2 (BL-2) conditions. Cryopreserved cells were thawed in a $37{ }^{\circ} \mathrm{C}$ water bath and cultured in Dulbecco's modified Eagle's medium (DMEM) plus $10 \%$ fetal bovine serum (FBS) supplemented with sodium pyruvate and L-glutamine in a tissue culture incubator in a $5 \%$ $\mathrm{CO}_{2}$ atmosphere. When the cells in culture reached about 70$90 \%$ confluence, they were harvested by treatment with trypsin-EDTA (0.25\% trypsin, $1 \mathrm{mM}$ EDTA) and washed with phosphate buffered saline (PBS, $\mathrm{pH}=7.4$ ). Finally, the cells were diluted in serum free medium (DMEM) and mixed with equal volumes of Matrigel (Corning, USA) for implantation. After a 7-day acclimatization period, an amount of 5 million A431 cells per animal suspended in $0.1 \mathrm{~mL}$ of medium was injected subcutaneously in the right hind flank region of the mouse using a syringe with a $26 \mathrm{G}$ hypodermic needle, taking care to avoid blood vessels. Successful implantation was indicated by the formation of a round, raised mass under the skin. The implanted mice were monitored for general health and tumor development daily.

Tumors were detectable around 1 week after implantation. The tumor size was measured with a calliper. When the A431 tumor were vascularized and had reached size of around $7 \pm 1$ $\mathrm{mm}$ in diameter, animals were randomly assigned into four groups of 5-6 animals each to either the vehicle control or the low $\left(60 \mathrm{mg} \mathrm{kg}^{-1}\right)$, medium $\left(90 \mathrm{mg} \mathrm{kg}^{-1}\right)$, or high $(120 \mathrm{mg}$ $\mathrm{kg}^{-1}$ ) dose F3-HYD groups.

The formulations were dosed subcutaneously around the scruff region of the animal once every day until the end of the study and the tumor sizes and mouse body weights monitored daily. Studies were continued either (a) until the predetermined end date indicated in the study design (50 days) or (b) until a maximal tumor size was reached or other humane end points (e.g., $\geq 15 \%$ body weight loss or signs of tumor necrosis) were reached, whichever occurred first.

Western Blotting. Once animals had been culled, tumors were excised, snap-frozen in liquid nitrogen, and stored at -80 ${ }^{\circ} \mathrm{C}$. For further analysis, the frozen tumors were ground into a fine powder $(200 \mathrm{mg})$ and suspended in a tissue protein extraction buffer (T-PER, $600 \mu \mathrm{L}$ ) supplemented with protease/phosphatase inhibitor cocktail $(6 \mu \mathrm{L})$ and ethylenediaminetetraacetic acid (EDTA, $0.5 \mathrm{M}, 6 \mu \mathrm{L}$ ). This mixture was homogenized until a uniform suspension was obtained ( $\sim 1 \mathrm{~min}$, on ice), and the samples were centrifuged at $13000 \mathrm{rpm}$ for $10 \mathrm{~min}$ at $4{ }^{\circ} \mathrm{C}$ (Hermle, Z323K, Wehingen, Germany). The supernatant was then transferred to a clean vial and the protein content of the samples estimated using the bicinchoninic acid (BCA) assay.

Samples containing $30 \mu \mathrm{g}$ of protein by BCA assay were mixed with lithium dodecyl sulfate (LDS) sample buffer (5 $\mu \mathrm{L}$ ), dithiothreitol (DTT, $2 \mu \mathrm{L}$ ), and water to make up the volume to $20 \mu \mathrm{L}$. Denatured samples $(20 \mu \mathrm{L})$ were loaded onto 4-12\% NuPAGE Novax Bis-Tris gels (Life Technologies, Paisley, U.K.) and subjected to electrophoresis in NuPAGE MOPS SDS running buffer in the Novex Minicell apparatus (Invitrogen, Waltham, MA, USA) according to the manufacturer's instructions. Protein standards (MagicMark XP Western standard) were included in the gel by following a method similar to that used for the preparation of the test samples. Proteins were transferred to Novex nitrocellulose membrane using the Novex Xcell II blot module (Invitrogen, Waltham, MA, USA) for $1.5 \mathrm{~h}$ at $30 \mathrm{~V}$.

Western blots were used to analyze the expression of actin (mouse monoclonal), a housekeeping protein, and acetylatedhistone 3 (Ac-H3, rabbit polyclonal); all steps were carried out at room temperature with gentle shaking. After $60 \mathrm{~min}$ in the blocking buffer (5\% BSA in PBS, $\mathrm{pH}=7.4)$, blots were incubated for $3 \mathrm{~h}$ with the following optimal dilutions of 
primary antibodies in the blocking buffer: 1 in 1000 for rabbit anti-human Ac-H3 (Abcam, Cambridge, U.K.) and 1 in 2000 for rabbit anti-human actin (Cell Signaling Technologies, Danvers, MA, USA). After a thorough washing in PBS containing 1\% Tween 20 (wash buffer), blots were incubated for $1.5 \mathrm{~h}$ in a 1 in 6000 dilutions of the second antibody in wash buffer, horseradish peroxidase (HRP)-conjugated antirabbit IgG (Cell Signaling Technologies, Danvers, MA, USA) for anti-Ac-H3 and HRP-conjugated goat anti-mouse IgG (Life Technologies, Paisley, U.K.) for anti-actin. Blots were developed by the SuperSignal West Pico Chemiluminescent substrate using the method described by the manufacturer (Thermo Scientific, Waltham, MA, USA).

\section{RESULTS}

Preparation of MET-CUDC-101 Formulations. $\mathrm{N}$ Palmitoyl- $N$-monomethyl- $N, N$-dimethyl- $N, N, N$-trimethyl-6-Oglycolchitosan (molecular envelope technology, MET) is a selfassembling chitosan derivative, ${ }^{16}$ which has a low critical micellar concentration $(1-20 \mu \mathrm{M})^{13,17}$ and forms stable selfassembled drug loaded nanoparticles. ${ }^{13}$ These nanoparticles significantly enhance drug bioavailability via the ocular, ${ }^{18}$ intravenous, $^{19,20}$ oral, ${ }^{13,21}$ and nose-to-brain ${ }^{22}$ routes. MET nanoparticles are mucoadhesive $\mathrm{e}^{13,21}$ and taken up via the oral route. ${ }^{21,23}$ In the current work we are exploring the delivery activity of MET nanoparticles via the subcutaneous route.

Two types of MET polymer (Table 1) were used for the encapsulation studies with different levels of palmitoylation and quaternary ammonium groups: MET1 with $14 \mathrm{~mol} \%$ palmitoylation and $26 \mathrm{~mol} \%$ quaternary ammonium groups; and MET2 with $5 \mathrm{~mol} \%$ palmitoylation and 5\% quaternary ammonium groups.

CUDC-101 (Figure 1b) was successfully encapsulated into the MET in all formulations F1-F3 (Figure 2a-c) using the method detailed above. The encapsulation involved both the ionic interaction between the anionic CUDC-101 and the cationic MET (formulations could only be prepared when ionized CUDC-101 was present as the sodium salt) and the hydrophobic interactions between CUDC-101's hydrophobic moieties and the palmitoyl groups of the MET (Figure 1a).

Three different MET-CUDC-101 formulations F1-F3 were prepared using two forms of MET polymer and polymer-drug ratios of 2:1 and 1:1 (Table 1). All three formulations formed uniform, spherical, and monodisperse nanoparticles with the size ranging from 100 to $200 \mathrm{~nm}$ (Figure 2a). For the preparation of freeze-dried formulations, addition of dextran as a cryoprotectant in the formulation made the reconstitution easier, presumably by preventing aggregation of the NPs during freeze-drying. Dextran is known to stabilize nanoparticles against aggregation during freeze-drying, although the exact mechanism remains unclear. ${ }^{24}$

The colloidal stability of the reconstituted MET-CUDC-101 formulations was assessed showing a clear effect of polymer chemistry on colloidal stability with the formulation F1 made with the polymer MET1 (14\% palmitoylation, $26 \%$ quaternary ammonium groups) proving to be less likely to sediment when compared to formulations F2 and F3 made with polymer MET2 (5\% palmitoylation, 5\% quaternary ammonium groups) (Figure 2b). Given the different substitution levels of these polymers, this suggests that both hydrophobicity and quaternary ammonium functions are required for stable MET-CUDC-101 binding, drug encapsulation, and colloidal a)

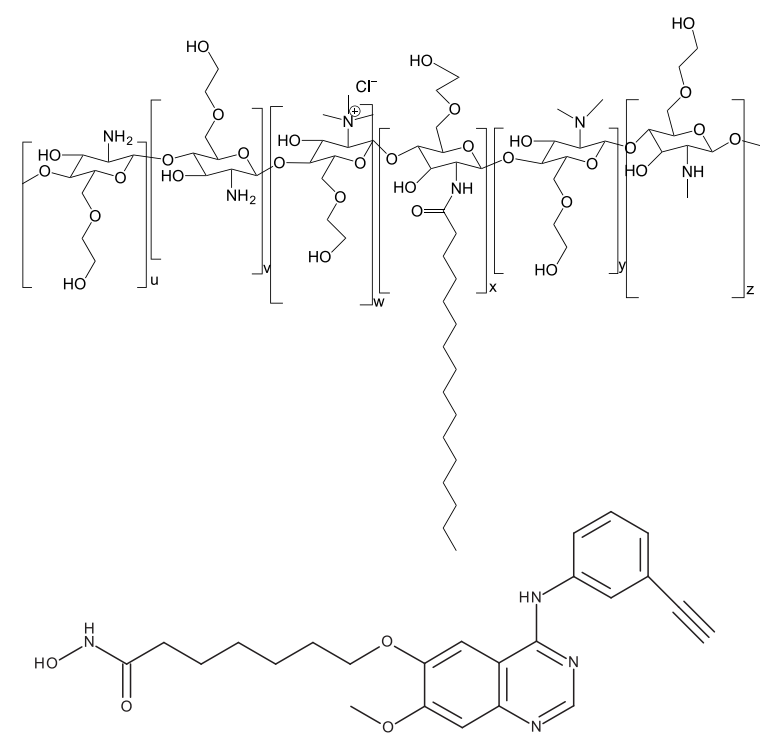

C)

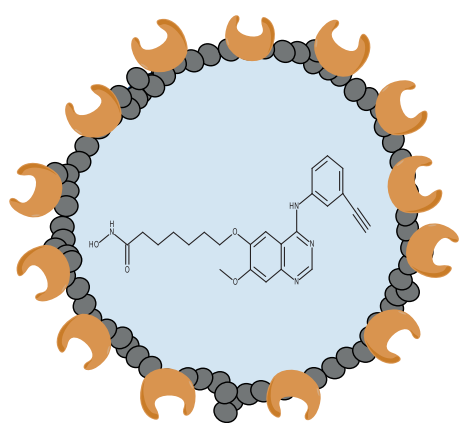

Figure 1. Chemical structures of polymer and drug: (a) $\mathrm{N}$-palmitoyl$\mathrm{N}$-monomethyl- $\mathrm{N}, \mathrm{N}$-dimethyl- $\mathrm{N}, \mathrm{N}, \mathrm{N}$-trimethyl-6-O-glycolchitosan (molecular envelope technology, MET); (b) CUDC-101 [7-(4-(3ethynylphenylamino)-7-methoxyquinazolin-6-yloxy)- $N$-hydroxyheptanamide] (NCBI, PubChem Database, CUDC-101, CID = 24756910); (c) schematic representation of CUDC-101 within a hyaluronidase coated polymer nanoparticle.

stability. Furthermore, a higher ratio of MET polymer to drug is found to improve stability as is evidenced by comparing MET2 formulations F2 (2:1 drug:polymer ratio) with F3 (1:1 drug:polymer ratio). There is no obvious difference between individual particles, e.g., in terms of appearance (TEM F2 vs F3; Figure 2a), yet a higher amount of polymer improves encapsulation, suggesting that subtle changes or equilibrium effects may be important here.

In order to achieve the higher drug loading required for clinical application, formulations were also freeze-dried and reconstituted at reduced volumes (Table 1). Only with F3 was a concentration of drug sufficient for potential clinical use as subcutaneous dosage form in humans achieved. F1 and F2 contained a higher concentration of the MET and could not be reconstituted to the target concentration of $50 \mathrm{mg} \mathrm{mL}^{-1}$ CUDC-101, as this would have involved a concentration of $100 \mathrm{mg} \mathrm{mL}^{-1}$ of the MET, at which the formulation was too viscous and lacked syringeability. For this reason, the study was progressed with F3.

To examine medium term stability of this formulation, the freeze-dried MET-CUDC-101 formulation F3 was stored at room temperature for up to 90 days and its stability during storage studied. Reconstitution of the dried formulation to 


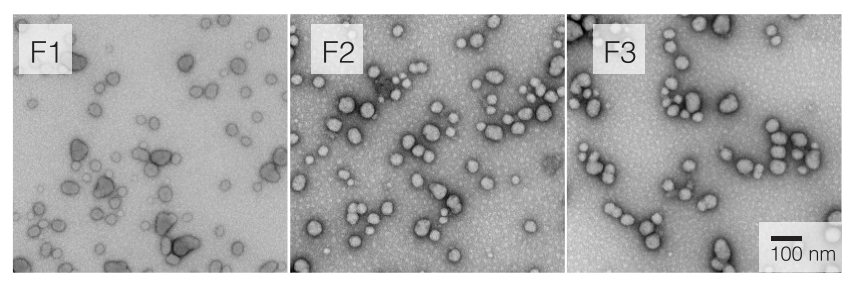

a)
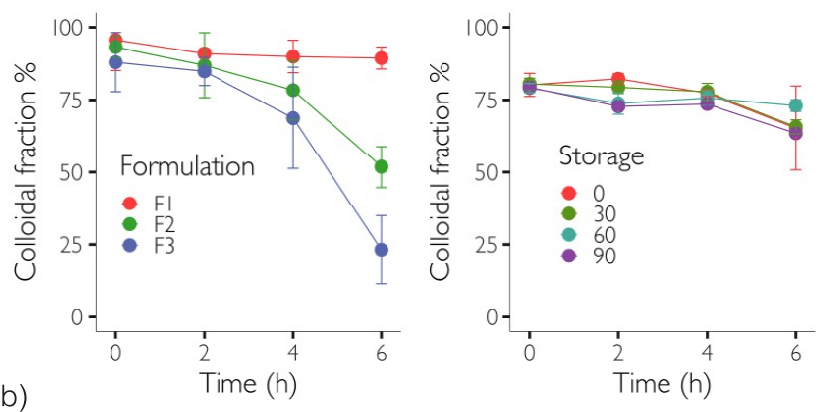

b)

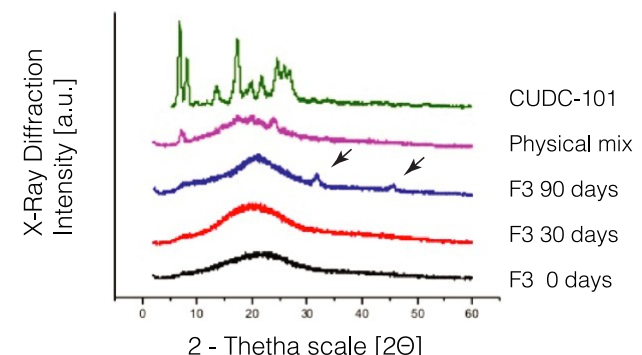

c)

Figure 2. Characterization of fresh and freeze-dried MET-CUDC-101 formulations: (a) TEM of formulations F1-F3; (b) colloidal stability of fresh formulations F1-F3 (5 mg mL ${ }^{-1}$ CUDC-101, left panel) and of the formulation F3 after freeze-drying, storage of up to 90 days, and reconstitution (50 mg mL $\mathrm{m}^{-1}$ CUDC-101, right panel); (c) X-ray diffraction analyses of stored freeze-dried samples of formulation F3 with arrows indicating $\mathrm{NaCl}$ peaks.

produce a dispersion containing $50 \mathrm{mg} \mathrm{mL}^{-1}$ CUDC-101 revealed that the formulation was stable for 3 months allowing reconstitution of colloidal NPs (Table 4) and resulting in

Table 4. Colloidal Properties on Reconstitution of Formulation F3 (50 mg mL $\mathrm{mL}^{-1}$ CUDC-101) after Storage

$\begin{array}{ccc}\begin{array}{c}\text { storage } \\ (\text { days })\end{array} & \begin{array}{c}z \text {-average diameter }(\mathrm{nm}) \\ (\text { mean } \pm \mathrm{sd}, n=3)\end{array} & \text { polydispersity } \\ 0 & 124 \pm 6 & 0.075 \pm 0.02 \\ 30 & 103 \pm 6 & 0.135 \pm 0.06 \\ 60 & 102 \pm 2 & 0.133 \pm 0.04 \\ 90 & 101 \pm 2 & 0.091 \pm 0.01\end{array}$

improved colloidal short-term stability, on reconstitution into a dispersion, compared to the fresh formulation (Figure 2) with $75-80 \%$ of the drug in the colloidal fraction.

We speculate that the balance of the drug was present as an indistinguishable sediment, although no sediment was actually observed (see below). Furthermore, after drying, the formulation seemed to sediment to a lesser extent than when freshly prepared. It should be noted that the concentration studied in the reconstituted freeze-dried formulation was 10 times as concentrated as the freshly prepared formulation. High concentrations of the MET are more viscous, ${ }^{16}$ and the viscosity of the disperse phase could have prevented sedimentation.

The dried formulation was amorphous throughout the 90day storage period, with the crystal peaks absent from the formulation but present in the drug-polymer mixture and the drug alone (Figure 2c). The XRD peaks seen in the 90-day sample correspond to those of the sodium chloride ${ }^{16}$ formed as a byproduct during the nanoparticle preparation. The fact that the CUDC-101 is in the amorphous form will confer some dissolution rate advantages and hence absorption advantages as the amorphous form of a drug normally has a higher dissolution rate than crystalline drug. ${ }^{25}$

Hyaluronidase-Coated MET-CUDC-101 Formulations. Surface coating of the MET-CUDC-101 nanoparticles with hyaluronidase (HYD) to produce HYD-MET-CUDC-101 nanoparticles was accomplished by reconstituting the freezedried MET-CUDC-101 F3 nanoparticles with a hyaluronidase solution.

The nanoparticles were characterized after freeze-drying and reconstitution (Figure 3a, Table 5). The surface coating (F3-
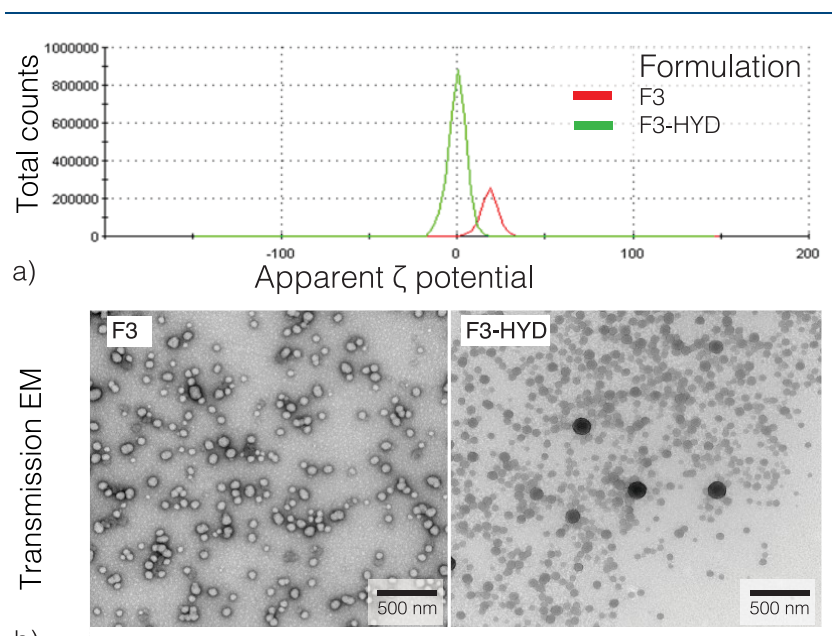

b)

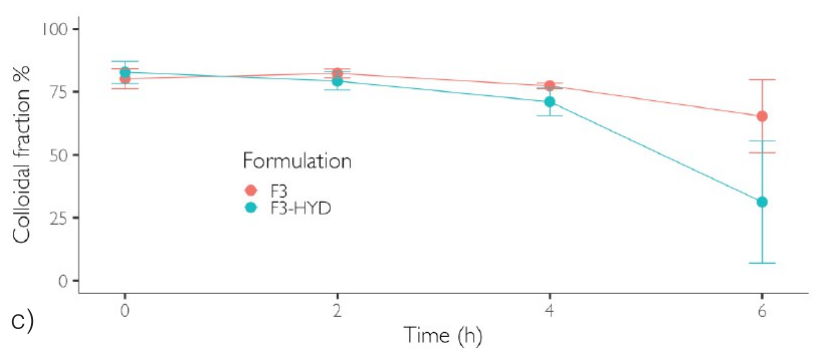

Figure 3. HYD-MET-CUDC-101 formulations: (a) $\zeta$ potential distribution of uncoated NP formulation F3 and HYD-coated F3HYD NPs [200 IU mL $\mathrm{mL}^{-1}\left(0.3 \mathrm{mg} \mathrm{mL}^{-1}\right)$ hyaluronidase], where values are significantly different from each other; $p<0.0001$ as determined by paired $t$ test, $n=3$; (b) TEM image with negative staining of F3 and of F3-HYD; (c) colloidal stability of MET-CUDC-101 formulation 3 after freeze-drying without (F3) and with hyaluronidase coating (F3-HYD).

HYD) was confirmed by the change in $\zeta$ potential from +18 $\mathrm{mV}$ to $0 \mathrm{mV}$ (Figure $3 \mathrm{a}$, Table 5 ), suggesting nonionic forces, e.g., steric and hydration forces, contributing to stabilization of the suspension. ${ }^{26}$

Vertebrate hyaluronidase has a $\mathrm{pI}$ of $5-6^{27}$ and will be close to $90 \%$ ionised (anionic) at the $\mathrm{pH}$ of the formulation $(\mathrm{pH}=$ 6.8). This slightly negative charge would facilitate the initial 
Table 5. MET-HYD-CUDC-101 Nanoparticles ${ }^{a}$

$\begin{array}{cccc}\text { formulation } & z \text {-average mean } \varnothing(\mathrm{nm}) & \text { polydispersity } & \zeta \text { potential }(\mathrm{mV}) \\ \text { F3 } & 195 \pm 6.0 & 0.143 \pm 0.016 & +18.4 \pm 1.5 \\ \text { F3-HYD } & 176 \pm 4.6 & 0.155 \pm 0.014 & -0.15 \pm 0.19^{b}\end{array}$

${ }^{a}$ All data are the mean \pm sd of $n=3 .^{b}$ Significantly different from MET-CUDC-101 formulation $3(p<0.0001)$.

adsorption onto the positively charged MET NPs. Consequently, the positive surface charge of the NPs would be neutralized and shielded leading to the neutral to anionic zeta potential observed (Figure 3a). Furthermore, the charge distribution graph reveals that only one charge population is present in the formulations, which provides solid evidence that the nanoparticles are surface coated with the enzyme hyaluronidase.

Coating of the NPs was further investigated by the size measurements of a number of F3-HYD formulations. Hyaluronidase isolated from bovine testes is a $61 \mathrm{kDa}$ enzyme with four subunits, which hydrolyzes the $\beta_{1 \rightarrow 4}$ glycosidic bonds in hyaluronan and chondroitin sulfate.

The predicted molecular size of globular proteins may be calculated by an in-built script in the Zetasizer software analysis package. Additionally an online calculator based on number of amino acid residues was also used ${ }^{28}$ for the estimation of the molecular size of hyaluronidase. The predicted size of hyaluronidase using both methods was $\sim 6$ $\mathrm{nm}$. The mean size of hyaluronidase analyzed by dynamic light scattering was measured to be $7 \mathrm{~nm}$ (by number and by volume), and the peak is seen in Figure 4. All F3-HYD formulations show the absence of the hyaluronidase peak (Figure 4a).

Gel electrophoresis showed the presence of some free enzyme for the F3-HYD formulation containing $10 \mathrm{mg} \mathrm{mL}^{-1}$ hyaluronidase (Figure $4 \mathrm{~b}$ ), an enzyme concentration that is 30 times that used in vivo. This is indicated by the visibly higher signal of the protein band found with free hyaluronidase compared to the same concentration of enzyme in the presence of nanoparticles and demonstrates that not all of the enzyme was free. These observations are also confirmed by densitometry of these bands showing relative higher peak height and bigger AUC (150.4 and 2565 vs 73.5 and 1305, respectively). No free enzyme was detected by the size distribution experiments however (Figure 4a). The gel electrophoresis method of enzyme detection is more sensitive than the size measurements. Taken together these experimental results (a drop in the positivity of the $\zeta$ potential, the absence of the free enzyme peak in the size distribution data, and the gel electrophoresis data) demonstrate that the particles were coated with hyaluronidase on injection.

The F3-HYD-MET-CUDC-101 nanoparticles appear dark in the negatively stained TEMs (Figure $3 b$, right panel) and appear to absorb the hydrophilic stain uniformly, which could indicate that hyaluronidase covers the surface of the nanoparticles. It should be noted that the MET-CUDC-101 F3 nanoparticles (Figure 3b, left panel) only have a typical dark exterior and a white interior as is normally seen with nanoparticles with hydrophobic interiors as the hydrophobic portions of the nanoparticles do not absorb the hydrophilic stain. $^{29,30}$

Given the change in $\zeta$ potential, the trend toward the enzyme destabilizing the nanoparticles is not surprising; however, the amount of drug in the colloidal fraction over
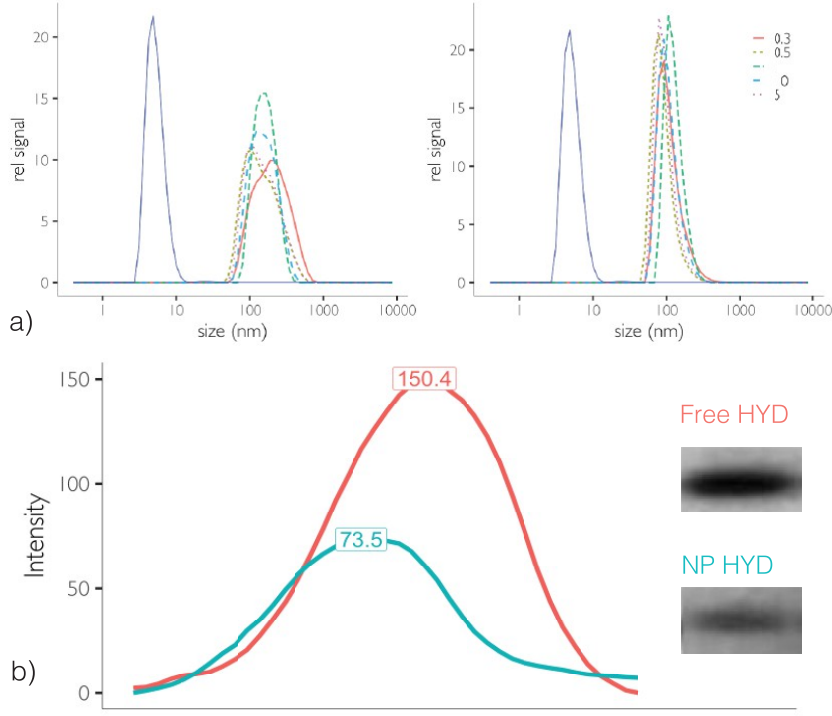

Figure 4. HYD-MET-CUDC-101 formulations. (a) Size distribution graphs of various formulations of F3-HYD loaded with CUDC-101 (10 $\mathrm{mg} \mathrm{mL}^{-1}$ CUDC-101). The numbers in the legend indicate the amount of hyaluronidase contained in $1 \mathrm{~mL}$ of the formulation. The concentration for hyaluronidase alone was $0.3 \mathrm{mg} \mathrm{mL}^{-1}$. Left panel: Volume size distribution graphs. Right panel: number size distribution graphs. Free hyaluronidase $\left(0.3 \mathrm{mg} \mathrm{mL}^{-1}\right.$, blue line $)$ is seen to have an average size of $\sim 7 \mathrm{~nm}$, and no free hyaluronidase is seen in the nanoparticle formulations. (b) Native protein gel comparing free hyaluronidase and F3-HYD. Both samples contain $10 \mathrm{mg} \mathrm{mL}^{-1}$ hyaluronidase. Samples containing less than $10 \mathrm{mg} \mathrm{mL}^{-1}$ hyaluronidase could not be analyzed by electrophoresis as a minimum protein content was required in each well. Densitometry plot of gels showing maximum relative intensity for free hyaluronidase (free HYD, 150.4) and nanoparticle loaded hyaluronidase (NP HYD, 73.5).

time was not significantly different when the nanoparticles were coated with the enzyme (Figure 3c).

Subcutaneous Absorption of CUDC-101 from METCUDC-101 Formulations. The pharmacokinetic study assessed plasma levels of the drug CUDC-101 and its main metabolite MET-M1 "M1" over $24 \mathrm{~h}$ after subcutaneous injection of concentrated formulations (F1, F2, F3) and formulation F3 coated with hyaluronidase (F3-HYD) in male Wistar rats (Figure 5).

Comparing the different formulations highlights a significant effect of NP formulation parameters on the shape of the concentration-time curves. Specifically, the use of the more hydrophobic MET1 polymer appears to lead to a much more sustained release profile compared to the particles made from the MET2 polymer with the last detectable concentration of drug: $127 \mathrm{ng} \mathrm{mL}^{-1}$ found after $8 \mathrm{~h}$ with MET1 vs $40 \mathrm{ng} \mathrm{mL}^{-1}$ after $7 \mathrm{~h}$ with MET2 (Table 6).

Bioavailability expressed as $\mathrm{AUC}_{0-24 \mathrm{~h}}$ for the different formulations shows statistically significant differences (Figure 5, Table 6). For example, preparation of formulation F1 with the more hydrophobic MET1 polymer results in doubling of the AUC (F1 2441 vs F2 $1232 \mathrm{ng} \mathrm{h} \mathrm{mL}^{-1}$ ) (Figure 5b, Table 6).

Furthermore, comparison of F2 and F3 (both prepared with MET2) suggests the administration of the more concentrated formulation with relatively lower amounts of polymer (polymer:drug ratios (w/w) of F2 1:1 and F3 1:1) given at the lower volume (F2, $2 \mathrm{~mL}$ of $25 \mathrm{mg} / \mathrm{mL}$ vs F3, $1 \mathrm{ml}$ of 50 


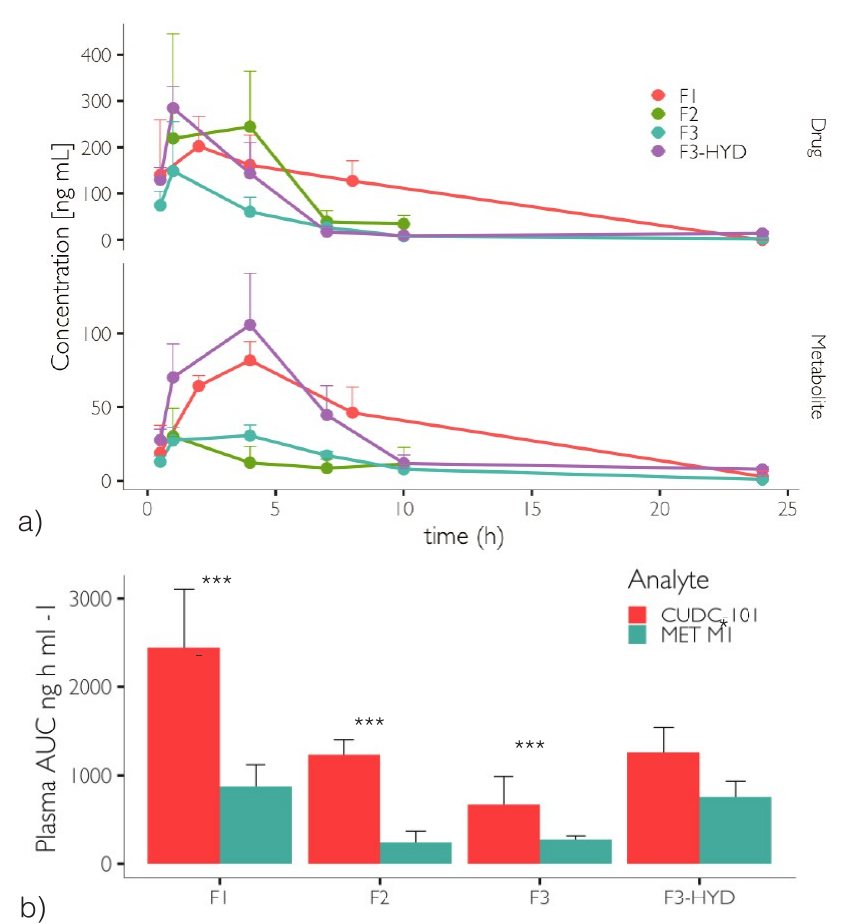

b)

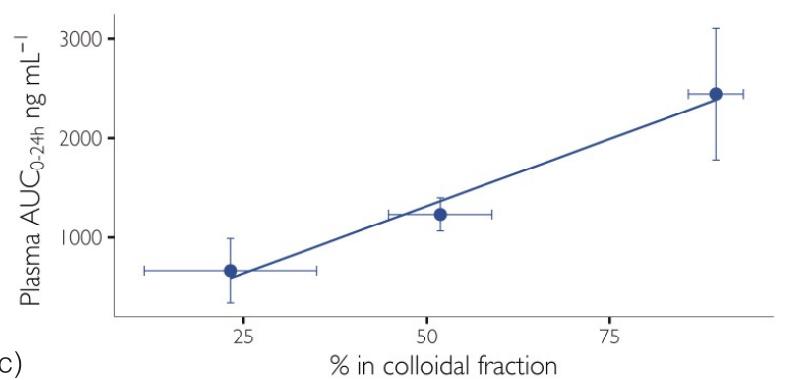

c)

Figure 5. Plasma drug and metabolite (a) levels (mean $\pm \mathrm{sd}, n=4-$ 5) following the subcutaneous administration of MET-CUDC-101 formulations to male Wistar rats $\left(50 \mathrm{mg} \mathrm{kg}^{-1}\right.$ CUDC-101). (b) Plasma $\mathrm{AUC}_{0-24}$ of CUDC-101 and metabolite Met M1 (*** indicates $p<0.001)$. The AUC values of F1-F3 are significantly different from each other (one-way ANOVA, using Prism 6.0c).* indicates $p<0.05$ significantly different from $\mathrm{F} 3$ alone (unpaired $t$ test). (c) Correlation between plasma $\mathrm{AUC}_{0-24}$ and $\%$ drug in the colloidal fraction $6 \mathrm{~h}$ after preparation of the NP dispersion: $\mathrm{AUC}_{0-24}$ $=27(\%$ colloidal drug $)-35.5, r^{2}=0.98$. $\mathrm{mg} / \mathrm{mL})$ cuts the bioavailability in half $\left(\mathrm{AUC}_{0-24 \mathrm{~h}} \mathrm{~F} 21232\right.$ vs F3 $667 \mathrm{ng} \mathrm{h} \mathrm{mL}^{-1}$ ).

In any case, higher AUCs tend to be linked to more sustained plasma levels, suggesting that absorption and transfer to the systemic circulation is a process that continues for several hours. One would expect this process to be dependent on the ability of the nanoparticles to retain the drug in the colloidal form.

The capacity of the different formulations F1-F3 to stabilize CUDC-101 in the form of nanoparticles can be expressed in a simplified form as the relative fraction of the original drug still present in colloidal form after, for example, $6 \mathrm{~h}$ (Figure 2).

Interestingly, if one plots for each formulation (F1-F3) the percentage of drug remaining in the colloidal form at this time point against the $\mathrm{AUC}_{0-24 \mathrm{~h}}$, a linear relationship is suggested (Figure 5c) with an $r^{2}$ for the correlation of 0.98 .

This finding suggests that sustained colloidal stability could be relevant for ensuring a high AUC; while this intuitively suggests facile sustained absorption, other factors ultimately could also contribute to these observations. Drug encapsulation is improved by the use of the more hydrophobic MET1 and as a result so is subcutaneous absorption (Figure $2 \mathrm{~d}$, Figure 5). The fact that the encapsulated drug is amorphous (Figure 2c) will also improve absorption. Finally, when the NPs of F3 are coated with hyaluronidase (F3-HYD), the bioavailability is restored with the $\mathrm{AUC}_{0-24 \mathrm{~h}}$ of this concentrated formulation being the same as that of F2 (1259

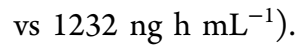

The drug metabolite levels also give an indication of which formulations are better absorbed. The time of maximal plasma concentration $\left(T_{\max }\right)$ of the drug CUDC-101 is typically reached more rapidly than that of the metabolite $M 1$, with the $C_{\max }$ of the metabolite being a fraction of the $C_{\max }$ of the drug. The $\mathrm{AUC}_{0-24 \mathrm{~h}}$ values follow similarly the trend seen with CUDC-101 and are highest for F1 $\left(868 \pm 252 \mathrm{ng} \mathrm{h} \mathrm{mL}^{-1}\right)$, with very similar values for F2 $\left(235 \pm 134 \mathrm{ng} \mathrm{h} \mathrm{mL} L^{-1}\right)$ and F3 $\left(276 \pm 40 \mathrm{ng} \mathrm{h} \mathrm{mL}^{-1}\right)$; however, the AUC for F3-HYD (751 \pm $\left.185 \mathrm{ng} \mathrm{h} \mathrm{mL}^{-1}\right)$ is much more similar in relative terms to $\mathrm{F} 1$ than is the case for CUDC-101 itself (Figure 5b and Table 6).

The AUCs of CUDC-101 and M1 are both increased in F1 (3.7X and $3.1 \times$, respectively) compared to the lowest AUC (F3) (Table 6, "AUC ${ }_{\mathrm{F} 3} x$-fold"). In contrast, for F3-HYD the relative increase is higher for the metabolite than the drug itself (1.9 vs 2.7), suggesting that in fact more drug has been delivered at earlier time points than is apparent from the

\section{Table 6. Pharmacokinetics of MET-CUDC-101 Formulations ${ }^{a}$}

\begin{tabular}{|c|c|c|c|c|}
\hline & \multicolumn{4}{|c|}{ formulation } \\
\hline & $\mathrm{F} 1$ & $\mathrm{~F} 2$ & F3 & F3-HYD \\
\hline \multicolumn{5}{|c|}{ CUDC-101 } \\
\hline $\mathrm{AUC}_{0-24 \mathrm{~h}}\left(\mathrm{ng} \mathrm{h} \mathrm{mL} \mathrm{m}^{-1}\right)$ & $2441 \pm 660^{* * *}$ & $1232 \pm 167^{* * * *}$ & $667 \pm 322 * * *$ & $1259 \pm 287^{*}$ \\
\hline$t_{\min }(\mathrm{h})^{b}$ & 8 & 7 & 4 & 4 \\
\hline$t_{\max }(\mathrm{h})$ & 2 & 4 & 1 & 1 \\
\hline$c\left(\mathrm{ng} \mathrm{mL}^{-1}\right)^{b}$ & 127 & 40 & 60 & 143 \\
\hline $\mathrm{AUC}_{\mathrm{F} 3}(x$-fold $)$ & 3.7 & 1.8 & 1.0 & 1.9 \\
\hline \multicolumn{5}{|c|}{ M1 } \\
\hline $\mathrm{AUC}_{0-24 \mathrm{~h}}\left(\mathrm{ng} \mathrm{h} \mathrm{mL} \mathrm{mL}^{-1}\right)$ & $868 \pm 252$ & $235 \pm 134$ & $276 \pm 40$ & $751 \pm 185$ \\
\hline$t_{\max }(\mathrm{h})$ & 4 & 1 & 4 & 4 \\
\hline $\operatorname{AUC}_{\mathrm{F} 3}(x$-fold $)$ & 3.1 & 0.8 & 1.0 & 2.7 \\
\hline
\end{tabular}

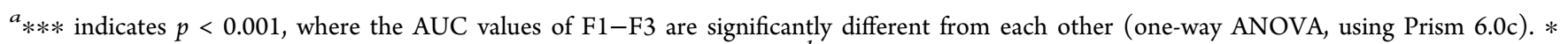
indicates $p<0.05$, significantly different from F3 alone (unpaired $t$ test). ${ }^{b} t_{\min }=$ final detectable concentration. 
available PK data alone. The metabolite data thus furthermore suggest that the more hydrophobic MET1 and coating with hyaluronidase could potentially lead to enhanced absorption.

Taken together, our data suggest that bioavailability and absorption of CUDC-101 are promoted by a more hydrophobic MET (MET 1) retaining CUDC-101 in a stable amorphous, colloidal form, a lower concentration and/or higher volume, and finally the presence of a hyaluronidase coating resulting in a ranking of relative bioavailability of $1 \times$ (F3), 1.8× (F2), 1.9× (HYD-F3), 3.7× (F1) (Table 6). It should be noted that we did not detect any irritability at the injection site, or in the relevant subcutaneous space, with this route of administration. As only F3 contained a sufficient concentration of drug to allow $500 \mathrm{mg}$ of CUDC-101 to be administered to humans, F3-HYD was studied in the therapeutic experiment.

Tumoricidal Activity of MET-CUDC-101 Formulations. The activity of the MET-CUDC-101 formulations was tested in a murine model using vascularised A431 flank xenograft tumors with animals being treated with once daily sc injections of CDUC-101 in MET nanoparticles. The formulation F3HYD was chosen because the coating with hyaluronidase dramatically increases bioavailability when compared to the uncoated F3; furthermore, due to the fact that administration volumes tend to be limiting for the subcutaneous route, the formulation with the higher drug loading when compared to F1 and F2 was selected. CUDC-101 could not be administered as a control as it is not water-soluble and a biocompatible solvent could not be found that would solubilize the drug to a concentration of $50 \mathrm{mg} \mathrm{mL}^{-1}$.

CUDC-101 is an inhibitor of histone deacetylase (HDAC), epidermal growth factor receptor (EGFR), and human epidermal growth factor receptor 2 (HER2). ${ }^{14}$ The HDAC inhibition may be probed by monitoring cellular levels of acetylated histone $\mathrm{H} 3$. Acetylated histone $\mathrm{H} 3$ was detected in tumor tissue following the subcutaneous administration of HYD-MET-CUDC-101 at a CUDC-101 dose of $60 \mathrm{mg} \mathrm{kg}^{-1}$, confirming that after subcutaneous administration in MET nanoparticles the drug engages with its target as expected (Figure 6a).

Our data compare favorably with tumoricidal data published on the preclinical evaluation of CUDC- $101^{14}$ and are in line with the clinical histone deacetylase inhibition observed in head and neck squamous cell cancer (HNSCC) patients.

We compared the effect of continued treatment with CUDC-101 using the F3-HYD formulation at three different dose levels $\left(60,90\right.$, and $\left.120 \mathrm{mg} \mathrm{kg}^{-1}\right)$ with individual injection volumes ranging from 30 to $60 \mu \mathrm{L}$. Treatment commenced once tumors were established and vascularized $(7 \pm 1 \mathrm{~mm})$ and continued up to day 50; animals were removed from the study and sacrificed earlier if tumors reached a maximum size of $12 \mathrm{~mm}$ in diameter, if the tumor showed signs of necrosis, or if the animals showed signs of toxicity (e.g., loss of body weight of $>15 \%$ ). The effect of treatment expressed as "survival probability” using a Kaplan-Meier diagram (Figure 6b) shows that all untreated animals were removed from the study within 15 days.

All animals treated with CUDC-101 in the F3-HYD formulation survived longer with median survival estimates increasing from 15 days for the untreated group to 18.5 (60 mg $\left.\mathrm{kg}^{-1}\right), 43\left(90 \mathrm{mg} \mathrm{kg}^{-1}\right)$, and 24 days $\left(120 \mathrm{mg} \mathrm{kg}^{-1}\right)$, in the best case almost tripling the time on study ("survival probability", Figure 6). The $90 \mathrm{mg} \mathrm{kg}^{-1}$ dose was superior to the 60 and 120

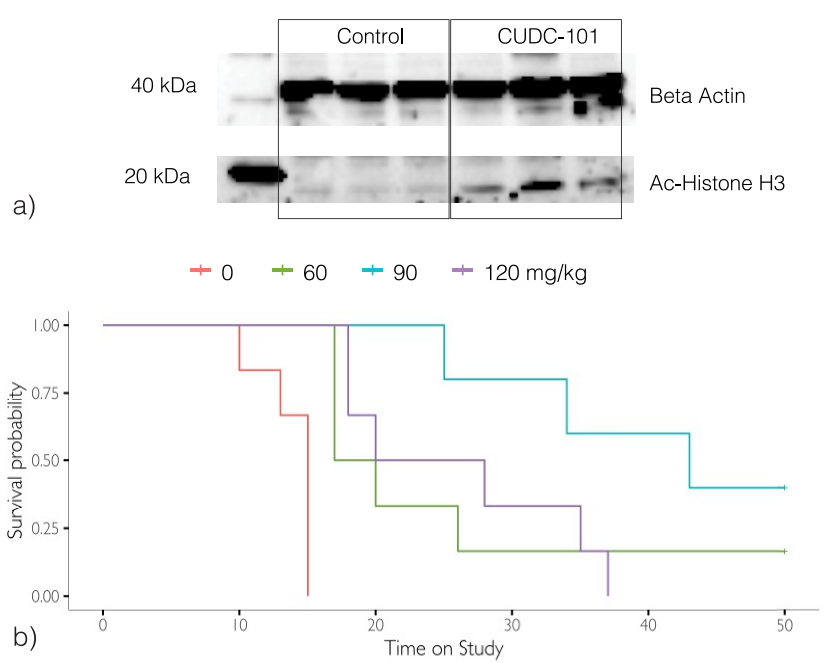

Figure 6. Pharmacological effects following the sc administration of F3-HYD-MET-CUDC-101 (60, 90, $120 \mathrm{mg} / \mathrm{kg}$ ). (a) Western blots showing the presence of acetylated histone (Ac-histone $\mathrm{H} 3$ ) in tumor samples isolated from an A431 mouse model $(n=3)$ following the subcutaneous injection of formulations at a CUDC-101 dose of $60 \mathrm{mg}$ $\mathrm{kg}^{-1}$ daily over 3 days. Tumor samples were isolated $6 \mathrm{~h}$ after administration of the last dose, and $\beta$-actin was used as an internal standard. (b) Kaplan-Meier survival plot $(n=6)$ of mice dosed daily from day 7 to the end of study. All treatment groups were significantly different from the control untreated group, $p<0.0001$ [log-rank (Mantel-Cox) test, using Prism software version 6.0c].

$\mathrm{mg} \mathrm{kg}^{-1}$ dose levels. The number of animals in the treatment groups being removed from the study for reasons other than progression of tumor growth (i.e., tumor necrosis or signs of toxicity) warrants a closer look at the individual cases (Figure 7): While all untreated animals ("0") were removed due to tumor progression, only three animals in the lowest dose treatment group ( $60 \mathrm{mg} \mathrm{kg}^{-1}$, “60") had tumors that reached the maximum size (Figure 7). All other treated animals in this group showed a marked delay and decrease in tumor growth with three animals showing complete regression at the end of the study.

Nevertheless, a large proportion of treated animals were removed before the study end (Figure 7) either because of signs of toxicity (e.g., loss of body weight loss) or because tumors started to show signs of central necrosis. About half of the animals in the highest dose group $\left(120 \mathrm{mg} \mathrm{kg}^{-1}\right)$ were euthanized because of signs of toxicity after prolonged treatment. While all the control group animals had to be euthanized by 15 days, three mice from the $90 \mathrm{mg} \mathrm{kg}^{-1}$ treatment group survived for at least 50 days.

\section{DISCUSSION}

The subcutaneous delivery of a high dose of hydrophobic drug remains challenging; a drug such as CUDC-101 requires a human parenteral (intravenous) dose of at least $500 \mathrm{mg}^{15}$ and has a low water solubility of $30 \mu \mathrm{g} \mathrm{mL}^{-1}$, making subcutaneous dosing particularly problematic. As data suggest that patients largely prefer subcutaneous dosage forms to intravenous dosage forms, ${ }^{3}$ we set out to prepare a subcutaneous dosage form from CUDC-101. By encapsulation in polymeric MET nanoparticles, we were able to incorporate $50 \mathrm{mg} \mathrm{mL}^{-1}$ of CUDC-101 in a water dispersible nanoenabled formulation (Table 1). This form will allow administration of $500 \mathrm{mg}$ of CUDC-101 as a subcutaneous injection of clinically acceptable 


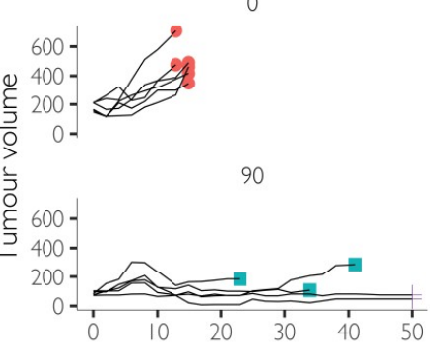

a)
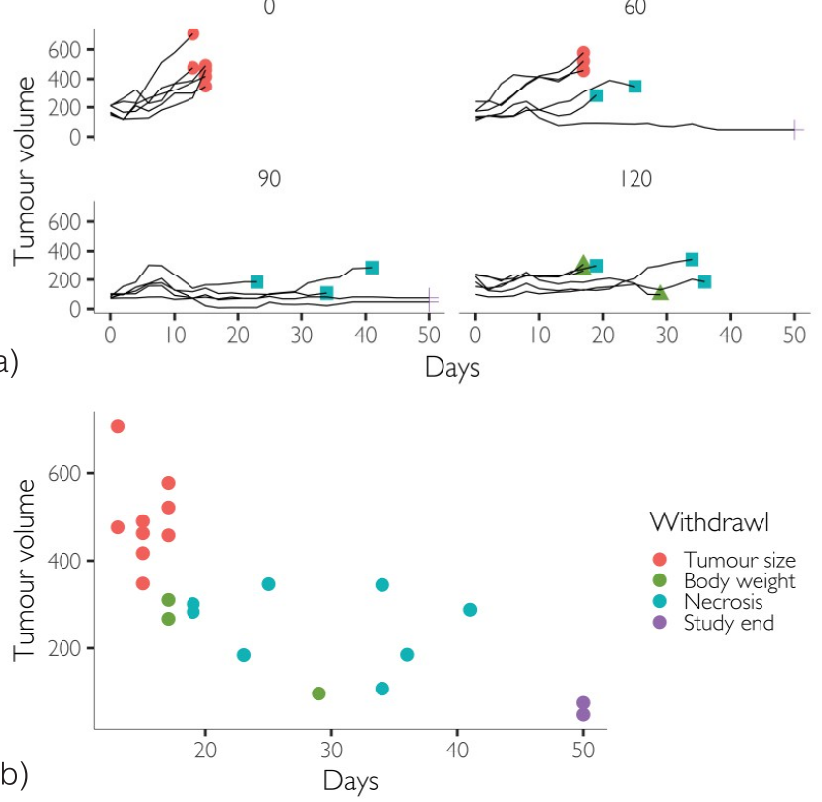

Figure 7. Effect of treatment at different dose levels of CUDC-101. (a) Panels show the growth of individual tumors (volume $\mathrm{mm}^{3}$ ) over time for each dose level of CUDC-101 (panels showing 0, 60, 90, 120 $\mathrm{mg} \mathrm{kg}^{-1}$ ) with reason and day of removal from the study indicated by final symbol and color (legend as in (b)). (b) Correlation of tumor size at removal vs time of removal from the study (removal reason shown by symbol and color).

volumes of 5-10 $\mathrm{mL}^{8,9}$ injected three times a week, thus effectively replacing the $1 \mathrm{~h}$ infusion given three times a week in the clinical study. ${ }^{15}$

Drug loading on to the MET polymer nanoparticles is achieved by a combination of electrostatic forces between the anionic CUDC-101 (ionized at alkaline $\mathrm{pH}$ ) and the cationic MET polymer which has a permanent quaternary ammonium positive charge ${ }^{16}$ and the hydrophobic attraction between the palmitoyl chains of the MET polymer and the hydrophobic moieties of CUDC-101. This conclusion is supported by the fact that an increase in the level of both quaternary ammonium and hydrophobic groups leads to more stable nanoparticles being formed (Figure 2c). This combination of interaction forces also allows encapsulation of amphotericin B (AmB) in MET nanoparticles, ${ }^{21}$ with electrostatic attraction between the anionic carboxylate function and the MET positively charged moiety and the interaction between the hydrophobic polyene groups on the drug and the palmitoyl groups contributing to the AmB encapsulation.

The hydrophobicity of amphiphilic MET polymers has been known to promote drug encapsulation, ${ }^{12,18}$ but the superior stability of F1 (MET1) compared to F2 (MET2) suggests that the polymers' hydrophobicity is also helping to retain a large proportion of the drug in colloidal form (Figure 2c).

Similarly, sufficient amounts of polymer relative to the drug loading improve colloidal stability (F2 2:1 ratio vs F3 1:1 ratio). Interestingly, freeze-drying appears to increase the stability of the MET-CUDC-101 formulation F3 (Figure 2) and allows longer term storage. The reason for the improved colloidal stability of the reconstituted F3 formulation over $6 \mathrm{~h}$ could be due to the relatively higher viscosity of the formulation caused by the presence of $50 \mathrm{mg} \mathrm{mL}^{-1}$ of the MET polymer. ${ }^{16}$

The normal subcutaneous injection volume is $1.5 \mathrm{~mL}$ for humans but can be increased using hyaluronidase which is known to degrade the interstitial matrix and thus enables fluid transfer to the subcutaneous space. ${ }^{6}$ However, the incorporation of active hyaluronidase is so far only possible in an aqueous formulation. ${ }^{6}$

To potentially enable the administration of clinically relevant doses of MET-CUDC-101 in volumes achievable in humans via the subcutaneous route, we utilized drug loaded MET nanoparticles coated with bovine hyaluronidase (Figure 3). This provides a convenient method of adding the enzyme to a formulation containing a hydrophobic drug. The hyaluronidase coating is verified by the change in $\zeta$ potential from a positive value $(+18 \mathrm{mV})$ to a neutral value $(-0.15)$ on addition of hyaluronidase (Figure 3a), the size distribution data (Figure $4 a)$, and the morphological changes seen with the darkened particles in the negatively stained TEMs which indicated that they are coated with a hydrophilic coating that absorbs the stain (Figure $3 b$ ). As we did not specifically remove unbound hyaluronidase, there is also likely to be hyaluronidase within the formulation disperse phase (e.g., Figure $4 \mathrm{~b}$ for high concentrations). The formulations are stable, when stored dried, for at least 3 months (Table 4 and Figure 3c).

Human recombinant hyaluronidase has been approved for the formulation of subcutaneous trastuzumab. ${ }^{6}$ Others have used hyaluronidase immobilized on silica nanoparticles and injected these with carboplatin peritumorally (in close proximity to the tumor) in preclinical studies and shown that the hyaluronidase coated silica nanoparticles promote tumor volume reduction. ${ }^{32}$

This is thus the first report of a hyaluronidase coating on the surface of drug carrying NPs. Coating the NPs with hyaluronidase means that the NPs can improve their own transport through the subcutaneous space.

In an aqueous environment the vast majority of the drug dose would be present as insoluble drug crystals (for F3 50 $\mathrm{mg} / \mathrm{mL}$ about $99.99 \%)$. For this reason, CUDC-101 cannot be subcutaneously administered without an appropriate formulation. From the pharmacokinetics data (Table 6 and Figure 5) it is evident that encapsulation within the MET nanoparticles facilitated drug absorption via the subcutaneous route. We conclude that a stable colloidal formulation in which the drug is in the amorphous state (Figure 2c) leads to superior plasma exposure (Figure $5 \mathrm{~b}$ ) and ultimately tumoricidal activity (Figure 6). The MET particles are known to promote absorption through the oral route, ${ }^{13,21,23}$ but this is the first report showing that the MET NPs also facilitate absorption along the subcutaneous route. A comparison of the pharmacokinetics of F3-HYD with CUDC-101 administered intravenously at a dose of $5 \mathrm{mg} \mathrm{kg}^{-1} 31$ shows that much higher AUCs are achievable (1028 vs $320 \mathrm{mg} \mathrm{mL}^{-1} \mathrm{~h}^{-1}$ ), albeit using the higher dose (Figure 8). The hyaluronidase coating enhanced plasma exposure, and it is likely that the hyaluronidase enabled the formulation to spread within the subcutaneous space as hyaluronidase hydrolyzes a number of biopolymers (hyaluronic acid and chondroitin) ${ }^{7}$ within the extracellular matrix. Hyaluronidase activity would result in the formulation occupying a higher surface area, within the subcutaneous cavity, and the net result is the higher plasma exposure observed (Figure 5a and Figure 5b). 


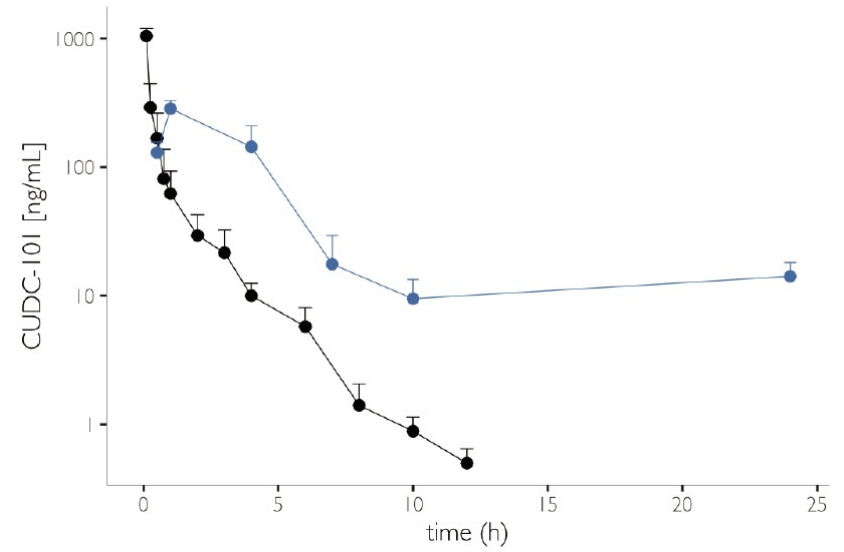

Figure 8. Comparison of pharmacokinetics of CUDC-101 given as intravenous injection at a dose of $5 \mathrm{mg} / \mathrm{kg}$ reported ${ }^{31}$ and subcutaneous F3-HYD-CUDC-101 at $50 \mathrm{mg} / \mathrm{kg}$.

CUDC-101 was designed as a drug that simultaneously but specifically inhibits multiple cancer-related molecular targets, namely, the receptor tyrosine kinases (RTK) EGFR and HER2 and the associated growth factor signaling pathway as well as histone deacetylase (HDAC), the latter an enzyme important in the epigenetic regulation of gene expression. ${ }^{14}$ Inhibition of HDAC reduces deacetylation of histone and non-histone substrates, for example, increasing acetylation of histone $\mathrm{H} 3$ and $\mathrm{H} 4$ and p53 and $\alpha$-tubulin. ${ }^{33}$ While it is not clear if the MET particles themselves are taken up from the subcutaneous space, our results confirm that the CUDC-101 in the formulation F3-HYD is able to reach the tumor and engage the relevant targets as shown by the acetylation of, for example, histone $\mathrm{H} 3$ (Figure 6a).

In addition, by inhibition of these targets, a range of other anticancer targets are engaged including Akt and MET RTKs, as well as full-length androgen receptor (flAR) and androgen receptor variant 7 (AR-V7). ${ }^{14,34}$ Taking into account the downstream signaling pathways, CUDC-101 is thus potentially active against a broad range of tumors, including NSLC, pancreatic, breast, glioblastoma, and prostate cancer.

The observation can be made that all animals that are taken off the study because of the increase in tumor volume are in the untreated group or the low dose group. All of those withdrawals occur for tumors larger than $400 \mathrm{~mm}^{3}$ and within the first 18 days. The F3-HYD formulation of CUDC-101 (90 $\mathrm{mg} \mathrm{kg}^{-1}$ ) prolonged the estimated median survival in a human A431 xenograft mouse model 3-fold, when compared to control (untreated) mice. The group that received $120 \mathrm{mg}$ $\mathrm{kg}^{-1}$ dose, which is also the maximum tolerated dose for CUDC-101 in mice, showed signs of toxicity in a proportion of animals after prolonged treatment (Figure 7).

A large proportion of animals treated with CUDC-101 were removed from the study early because they developed superficial necrosis of the xenograft tumors; 2/6 (60 mg $\left.\mathrm{kg}^{-1}\right), 3 / 5\left(90 \mathrm{mg} \mathrm{kg}{ }^{-1}\right)$, and $3 / 3\left(120 \mathrm{mg} / \mathrm{kg}^{-1}\right)$ animals developed necrosis from day 19 onward (Figure 7 ).

Tumor necrosis is thought to develop when the nutrient supply to the central core cannot keep up with the speed of development/growth. Typically, one would see necrosis when tumors grow too fast for the blood supply to keep up so that central areas are undersupplied. However, a closer look at the timing and size of tumors suggests that this is not the case as no necrosis was observed in any of the animals in the largest and most rapidly growing tumors in the control group (dose of " 0 ", Figure 7). In fact, it appears that tumors of intermediate size, i.e., that have been treated but have not shown a complete response, are most likely to develop signs of necrosis (Figure 7). This would suggest a potential effect associated with CUDC-101 pharmacological activity and a number of potential pathways could lead to antiangiogenic effects with evidence for both the HDAC and EGFR elements potentially being involved:

- Initial studies of CUDC-101 pharmacology revealed hypoxia inducible factor (HIF) $1 \alpha$ downregulation by CUDC-101, an effect also described for other HDAC inhibitors. $^{14,33}$

- Furthermore, EGFR inhibition has been linked to downregulation of vascular endothelial growth factor (VEGF). ${ }^{35}$ Taken together, our data support the notion that the antiproliferative effects of CUDC-101 at least in part may be linked to its antiangiogenic activity.

Interestingly, we were able to show activity in a tumor bearing model with a subcutaneous dose of $90 \mathrm{mg} \mathrm{kg}^{-1}$ (Figure 6), whereas previous preclinical work on CUDC-101 formulations administered via the intravenous and intraperitoneal route reports tumoricidal activity with an intravenous or peritoneal dose of $120 \mathrm{mg} \mathrm{kg}{ }^{-1} \cdot{ }^{14}$ Our data suggest that the HYD-MET-CUDC-101 formulation may be more effective than the original formulation (formulated with cyclodextrin) used in earlier preclinical studies. ${ }^{14}$ Target engagement in the form of deacetylated histone $\mathrm{H} 3$ is shown after three subcutaneous doses of $60 \mathrm{mg} \mathrm{kg}^{-1}$ in the A431 tumor xenografts (Figure 6a) with previous work demonstrating this in the HCC-827 and $\mathrm{H} 292$ xenografts after three daily doses of intravenous or intraperitoneal injections (the exact route of administration is not stated by the authors) of CUDC101 at $30 \mathrm{mg} \mathrm{kg}^{-1}$ (HC-827) and $60 \mathrm{mg} \mathrm{kg}^{-1}$ (H292). ${ }^{14}$

\section{CONCLUSION}

In this work we offer a solution to a pharmaceutical formulation problem: that of having to deliver a high dose of a hydrophobic drug (and as a result a large aqueous volume) through the subcutaneous route. Encapsulation of the drug in nanoparticles increased the aqueous incorporation over 1000fold (from about $30 \mu \mathrm{g} \mathrm{mL}^{-1}$ to $50 \mathrm{mg} \mathrm{mL}^{-1}$ ). Once an aqueous disperse phase could be used, the high volume needed to administer the dose was administered using a hyaluronidase coating on the drug loaded NPs. Ultimately drug encapsulation directly controlled drug plasma exposure, and tumoricidal activity in a preclinical model was obtained with the subcutaneous hyaluronidase coated NPs. This is the first report of hyaluronidase coated and drug loaded NPs being used to deliver chemotherapy in vivo.

\section{AUTHOR INFORMATION}

\section{Corresponding Author}

Andreas G. Schätzlein - UCL School of Pharmacy, London WC1N 1AX, U.K.; Nanomerics Ltd., London EC2Y 5AU,

U.K.; ๑ orcid.org/0000-0003-3907-6603;

Email: a.schatzlein@ucl.ac.uk

\section{Authors}

Ramesh Soundararajan - UCL School of Pharmacy, London WCIN 1AX, U.K. 
George Wang - UCL School of Pharmacy, London WC1N IAX, U.K.

Asya Petkova - UCL School of Pharmacy, London WC1N IAX, U.K.

Ijeoma F. Uchegbu - UCL School of Pharmacy, London WC1N 1AX, U.K.; Nanomerics Ltd., London EC2Y 5AU, U.K.

Complete contact information is available at:

https://pubs.acs.org/10.1021/acs.molpharmaceut.0c00294

\section{Notes}

The authors declare no competing financial interest.

\section{ACKNOWLEDGMENTS}

Curis Inc. is acknowledged for partially funding this work.

\section{REFERENCES}

(1) Moreau, P.; Pylypenko, H.; Grosicki, S.; Karamanesht, I.; Leleu, X.; Grishunina, M.; Rekhtman, G.; Masliak, Z.; Robak, T.; Shubina, A.; Arnulf, B.; Kropff, M.; Cavet, J.; Esseltine, D.-L.; Feng, H.; Girgis, S.; van de Velde, H.; Deraedt, W.; Harousseau, J.-L. Subcutaneous Versus Intravenous Administration of Bortezomib in Patients with Relapsed Multiple Myeloma: a Randomised, Phase 3, Non-Inferiority Study. Lancet Oncol. 2011, 12 (5), 431-440.

(2) De Sio, L.; Mariani, G.; Muzzucconi, M. G.; Chistolini, A.; Tirindelli, M. C.; Mandelli, F. Comparison Between Subcutaneous and Intravenous DDAVP in Mild and Moderate Hemophilia a. Thromb. Haemostasis 1985, 54 (2), 387-389.

(3) Jin, J.-F.; Zhu, L.-L.; Chen, M.; Xu, H.-M.; Wang, H.-F.; Feng, X.-Q.; Zhu, X.-P.; Zhou, Q. The Optimal Choice of Medication Administration Route Regarding Intravenous, Intramuscular, and Subcutaneous Injection. Patient Prefer Adherence 2015, 9, 923-942.

(4) Mathaes, R.; Koulov, A.; Joerg, S.; Mahler, H.-C. Subcutaneous Injection Volume of Biopharmaceuticals-Pushing the Boundaries. J. Pharm. Sci. 2016, 105 (8), 2255-2259.

(5) Richter, W. F.; Bhansali, S. G.; Morris, M. E. Mechanistic Determinants of Biotherapeutics Absorption Following SC Administration. AAPS J. 2012, 14 (3), 559-570.

(6) Dunn, A. L.; Heavner, J. E.; Racz, G.; Day, M. Hyaluronidase: a Review of Approved Formulations, Indications and Off-Label Use in Chronic Pain Management. Expert Opin. Biol. Ther. 2010, 10 (1), 127-131.

(7) Stern, R.; Jedrzejas, M. J. Hyaluronidases: Their Genomics, Structures, and Mechanisms of Action. Chem. Rev. 2006, 106 (3), $818-839$.

(8) Ismael, G.; Hegg, R.; Muehlbauer, S.; Heinzmann, D.; Lum, B.; Kim, S.-B.; Pienkowski, T.; Lichinitser, M.; Semiglazov, V.; Melichar, B.; Jackisch, C. Subcutaneous Versus Intravenous Administration of (Neo)Adjuvant Trastuzumab in Patients with HER2-Positive, Clinical Stage I-III Breast Cancer (HannaH Study): a Phase 3, Open-Label, Multicentre, Randomised Trial. Lancet Oncol. 2012, 13 (9), 869-878.

(9) Davies, A.; Berge, C.; Boehnke, A.; Dadabhoy, A.; Lugtenburg, P.; Rule, S.; Rummel, M.; McIntyre, C.; Smith, R.; Badoux, X. Subcutaneous Rituximab for the Treatment of B-Cell Hematologic Malignancies: a Review of the Scientific Rationale and Clinical Development. Adv. Ther. 2017, 34 (10), 2210-2231.

(10) Williams, H. D.; Trevaskis, N. L.; Charman, S. A.; Shanker, R. M.; Charman, W. N.; Pouton, C. W.; Porter, C. J. H. Strategies to Address Low Drug Solubility in Discovery and Development. Pharmacol. Rev. 2013, 65 (1), 315-499.

(11) Kalepu, S.; Nekkanti, V. Insoluble Drug Delivery Strategies: Review of Recent Advances and Business Prospects. Acta Pharm. Sin. B 2015, 5 (5), 442-453.

(12) Le, H. T. B.; Schatzlein, A. G.; Uchegbu, I. F. Polymer Hydrophobicity Has a Positive Effect on the Oral Absorption of Cyclosporine a From Poly(Ethylenimine) Based Nanomedicines. Pharm. Nanotechnol. 2013, 1, 15-25.
(13) Siew, A.; Le, H.; Thiovolet, M.; Gellert, P.; Schatzlein, A.; Uchegbu, I. Enhanced Oral Absorption of Hydrophobic and Hydrophilic Drugs Using Quaternary Ammonium Palmitoyl Glycol Chitosan Nanoparticles. Mol. Pharmaceutics 2012, 9 (1), 14-28.

(14) Lai, C.-J.; Bao, R.; Tao, X.; Wang, J.; Atoyan, R.; Qu, H.; Wang, D.-G.; Yin, L.; Samson, M.; Forrester, J.; Zifcak, B.; Xu, G.-X.; DellaRocca, S.; Zhai, H.-X.; Cai, X.; Munger, W. E.; Keegan, M.; Pepicelli, C. V.; Qian, C. CUDC-101, a Multitargeted Inhibitor of Histone Deacetylase, Epidermal Growth Factor Receptor, and Human Epidermal Growth Factor Receptor 2, Exerts Potent Anticancer Activity. Cancer Res. 2010, 70 (9), 3647-3656.

(15) Galloway, T. J.; Wirth, L. J.; Colevas, A. D.; Gilbert, J.; Bauman, J. E.; Saba, N. F.; Raben, D.; Mehra, R.; Ma, A. W.; Atoyan, R.; Wang, J.; Burtness, B.; Jimeno, A. A Phase I Study of CUDC-101, a Multitarget Inhibitor of HDACs, EGFR, and HER2, in Combination with Chemoradiation in Patients with Head and Neck Squamous Cell Carcinoma. Clin. Cancer Res. 2015, 21 (7), 1566-1573.

(16) Chooi, K. W.; Simão Carlos, M. I.; Soundararajan, R.; Gaisford, S.; Arifin, N.; Schätzlein, A. G.; Uchegbu, I. F. Physical Characterisation and Long-Term Stability Studies on Quaternary Ammonium Palmitoyl Glycol Chitosan (GCPQ)-A New Drug Delivery Polymer. J. Pharm. Sci. 2014, 103 (8), 2296-2306.

(17) Odunze, U.; O’Brien, F.; Godfrey, L.; Schätzlein, A.; Uchegbu, I. Unusual Enthalpy Driven Self Assembly at Room Temperature with Chitosan Amphiphiles. Pharm. Nanotechnol. 2019, 7 (1), 57-71.

(18) Qu, X.; Khutoryanskiy, V. V.; Stewart, A.; Rahman, S.; Papahadjopoulos-Sternberg, B.; Dufes, C.; McCarthy, D.; Wilson, C. G.; Lyons, R.; Carter, K. C.; Schätzlein, A.; Uchegbu, I. F. Carbohydrate-Based Micelle Clusters Which Enhance Hydrophobic Drug Bioavailability by Up to 1 Order of Magnitude. Biomacromolecules 2006, 7 (12), 3452-3459.

(19) Fisusi, F. A.; Siew, A.; Chooi, K. W.; Okubanjo, O.; Garrett, N.; Lalatsa, K.; Serrano, D.; Summers, I.; Moger, J.; Stapleton, P.; SatchiFainaro, R.; Schatzlein, A. G.; Uchegbu, I. F. Lomustine Nanoparticles Enable Both Bone Marrow Sparing and High Brain Drug Levels - a Strategy for Brain Cancer Treatments. Pharm. Res. 2016, 33 (5), $1289-1303$.

(20) Lalatsa, A.; Schätzlein, A. G.; Garrett, N. L.; Moger, J.; Briggs, M.; Godfrey, L.; Iannitelli, A.; Freeman, J.; Uchegbu, I. F. Chitosan Amphiphile Coating of Peptide Nanofibres Reduces Liver Uptake and Delivers the Peptide to the Brain on Intravenous Administration. J. Controlled Release 2015, 197, 87-96.

(21) Serrano, D. R.; Lalatsa, A.; Dea-Ayuela, M. A.; Bilbao-Ramos, P. E.; Garrett, N. L.; Moger, J.; Guarro, J.; Capilla, J.; Ballesteros, M. P.; Schätzlein, A. G.; Bolás, F.; Torrado, J. J.; Uchegbu, I. F. Oral Particle Uptake and Organ Targeting Drives the Activity of Amphotericin B Nanoparticles. Mol. Pharmaceutics 2015, 12 (2), 420-431.

(22) Godfrey, L.; Iannitelli, A.; Garrett, N. L.; Moger, J.; Imbert, I.; King, T.; Porreca, F.; Soundararajan, R.; Lalatsa, A.; Schatzlein, A. G.; Uchegbu, I. F. Nanoparticulate Peptide Delivery Exclusively to the Brain Produces Tolerance Free Analgesia. J. Controlled Release 2018, 270, 135-144.

(23) Garrett, N. L.; Lalatsa, A.; Uchegbu, I.; Schätzlein, A.; Moger, J. Exploring Uptake Mechanisms of Oral Nanomedicines Using Multimodal Nonlinear Optical Microscopy. J. Biophoton. 2012, 5 (5-6), 458-468.

(24) Hinrichs, W. L. J.; Manceñido, F. A.; Sanders, N. N.; Braeckmans, K.; De Smedt, S. C.; Demeester, J.; Frijlink, H. W. The Choice of a Suitable Oligosaccharide to Prevent Aggregation of PEGylated Nanoparticles During Freeze Thawing and Freeze Drying. Int. J. Pharm. 2006, 311 (1-2), 237-244.

(25) Hancock, B. C.; Parks, M. What Is the True Solubility Advantage for Amorphous Pharmaceuticals? Pharm. Res. 2000, 17 (4), 397-404.

(26) Santander-Ortega, M. J.; de la Fuente, M.; Lozano, M. V.; Bekheet, M. E.; Progatzky, F.; Elouzi, A.; Uchegbu, I. F.; Schätzlein, A. G. Hydration Forces as a Tool for the Optimization of Core-Shell Nanoparticle Vectors for Cancer Gene Therapy. Soft Matter 2012, 8 (48), 12080-12092. 
(27) MALMGREN, H. Characteristics of Testicular Hyaluronidase. Biochim. Biophys. Acta 1953, 11 (4), 524-529.

(28) CalcTool. Protein Size Calculator. 2008.

(29) Wang, W.; McConaghy, A. M.; Tetley, L.; Uchegbu, I. F. Controls on Polymer Molecular Weight May Be Used to Control the Size of Palmitoyl Glycol Chitosan Polymeric Vesicles. Langmuir 2001, 17 (3), 631-636.

(30) Wang, W.; Qu, X.; Gray, A. I.; Tetley, L.; Uchegbu, I. F. SelfAssembly of Cetyl Linear Polyethylenimine to Give Micelles, Vesicles, and Dense Nanoparticles. Macromolecules 2004, 37 (24), 9114-9122.

(31) Zhang, Q.; Wen, C.; Xiang, Z.; Ma, J.; Wang, X. Determination of CUDC-101 in Rat Plasma by Liquid Chromatography Mass Spectrometry and Its Application to a Pharmacokinetic Study. J. Pharm. Biomed. Anal. 2014, 90, 134-138.

(32) Scodeller, P.; Catalano, P. N.; Salguero, N.; Duran, H.; Wolosiuk, A.; Soler-Illia, G. J. A. A. Hyaluronan Degrading Silica Nanoparticles for Skin Cancer Therapy. Nanoscale 2013, 5 (20), 9690-9698.

(33) Ellis, L.; Hammers, H.; Pili, R. Targeting Tumor Angiogenesis with Histone Deacetylase Inhibitors. Cancer Lett. 2009, 280 (2), 145153.

(34) Sun, H.; Mediwala, S. N.; Szafran, A. T.; Mancini, M. A.; Marcelli, M. CUDC-101, a Novel Inhibitor of Full-Length Androgen Receptor (flAR) and Androgen Receptor Variant 7 (AR-V7) Activity: Mechanism of Action and in Vivo Efficacy. Horm. Cancer 2016, 7 (3), 196-210.

(35) van Cruijsen, H.; Giaccone, G.; Hoekman, K. Epidermal Growth Factor Receptor and Angiogenesis: Opportunities for Combined Anticancer Strategies. Int. J. Cancer 2005, 117 (6), 883888. 